J. Clin. Chem. Clin. Biochem.

Vol. 15,1977 , pp. 323-328

\title{
Phenolphthalein-monophosphat als Substrat zur Bestimmung der sauren Prostataphosphatase: Kritische Anmerkungen
}

\author{
Von H. Gallati \\ Diagnostische Forschungsabteilung F. Hoffmann-La Roche \& Co. AG, Basel/Schweiz
}

(Eingegangen am 10. September/28. November 1976)

Zusammenfassung: Albumin bindet Phenolphthalein im alkalischen Meßbereich von pH 10. Das Resonanzsystem dieses Säure-Basen-Indikators kann dadurch nicht vollständig ausgebildet werden, was zu einer entsprechenden Verminderung der Farbintensität führt.

Albumin bindet auch Phenolphthalein-monophosphat im pH-Bereich der enzymatischen Reaktion von pH 4-8. Dadurch wird je nach Konzentrationsverhältnis die Substratkonzentration entweder suboptimal, was sich in einer geringeren katalytischen Konzentration der Phosphatase zeigt, oder der Substratüberschuß wird vom Albumin gebunden, wodurch die Substrathemmung der Phosphatase verringert oder aufgehoben wird.

Durch Alkoholzusatz kann die Bindung des Phenolphthaleins wie auch des Phenolphthalein-monophosphats an Albumin zum Teil gelöst werden.

Die Resultate dieser Arbeit zeigen, daß die Aktivitätsbestimmung der Prostataphosphatase mit Phenolphthaleinmonophosphat als Substrat von verschiedenen Faktoren wesentlich beeinflußt wird, so daß eine Interpretation der gemessenen Farbintensität schwierig und eine Berechnung der katalytischen Konzentration auf Grund der erhaltenen Resultate häufig zu fehlerhaften Ergebnissen führt.

Critical observations on the use of phenolphthalein monophosphate as a substrate for the determination of prostatic acid phosphatase

Summary: At pH 10, which is in the alkaline measurement range, phenolphthalein is bound by albumin. The resonance system of this acid-base indicator cannot therefore be completely formed, and the colour intensity is correspondingly lower.

Albumin also binds phenolphthalein monophosphate at $\mathrm{pH} 4-8$, in the $\mathrm{pH}$ range of the enzymic reaction. Depending on the relative concentrations, the substrate concentration is therefore either suboptimal (manifested as a lower catalytic concentration of phosphatase), or the substrate excess is bound by albumin, so that the substrate inhibition of the phosphatase is decreased or abolished.

Binding of phenolphthalein and its monophosphate to albumin is partly prevented by the addition of alcohol.

These results show that the determination of prostate phosphatase with the substrate phenolphthalein monophosphate is markedly influenced by various factors. The interpretation of the observed colour intensity is therefore difficult, and the calculation of the catalytic concentration is frequently subject to error.

\section{Einfinhrung}

In einer vorangehenden Arbeit wurde dargelegt, dạß die saure Prostataphosphatase bei Anwesenheit höherer Alkohole (1-Butanol und 1:Pentanol) wesentlich aktiviert wird (1). Diese Aktivierung ist prinzipiell von der Art des eingesetzten Substrats unabhängig. Bei unseren dies- bezüglichen Versuchen mit den Substraten Phenolphthaleinphosphat und Thymolphthaleinphosphat wurde aber deutlich, daß die photometrisch gemessene Farbintensität des freigesetzten Chromophors neben der vorhandenen katalytischen Phosphatasekonzentration auch wesentlich von der Proteinkonzentration und anderen Zusätzen in der Testlösung abhängig ist. In dieser Arbeit 
wird der Einfluß von Protein und Alkohol auf die Farbintensität des Phenolphthaleins sowie auf die MichaelisKonstante des Phenolphthalein-monophosphats für saure Prostataphosphatase untersucht und diskutiert.

\section{Material und Methoden}

Die Chemikalien waren von analytischer Reinheit und wurden von Merck bezogen, außer Rinderserum-Albumin (Fluka), Phenolphthalein und Phenolphthalein-monophosphat (Sigma).

Die saure Prostataphosphatase (EC 3.1.3.2) wurde nach beschriebener Methode (1) aus menschlichem Prostatagewebe extrahiert, der pH-Wert der Enzymlösung auf 4,5 gesenkt und das ausgefällte Fremdprotein abzentrifugiert. Anschließend wurde die Prostataphosphatase auf CM-Cellulose und Sephadex G-150 chromatographiert. Die spezifische Aktivität der gereinigten Prostataphosphatase betrug $500 \mathrm{U} / \mathrm{mg}$ Protein. (Bestimmung der Phosphatase nach der konventionellen Testmethode (2)).

Zur Bestimmung der Prostataphosphatase wurden $0,1 \mathrm{ml}$ Enzymlösung zu $0,5 \mathrm{ml}$ vorgewärmter Substratpufferlösung zugemischt und während $30 \mathrm{~min}$ bei $37^{\circ} \mathrm{C}$ inkubiert. Die enzymatische Reaktion wurde durch Zugabe von $2,5 \mathrm{ml} \mathrm{0,4} \mathrm{mol} / 1$ Natriumphosphatpuffer vom pH 10 abgestoppt und die Farbintensität des freigesetzten Phenolphthaleins bei der Wellenlänge $546 \mathrm{~nm}$ photometrisch bestimmt.

Bei der Darstellung der Resultate wurde auf die Umrechnung der gemessenen Absorptionsdifferenz $\left(\Delta \mathbf{A}_{546 \mathrm{~nm}} / 30 \mathrm{~min}\right)$ in katalytische Konzentrationen (U/l) verzichtet, da die Farbintensität des freigesetzten Phenolphthaleins neben der Phosphatasekonzentration auch wesentlich von der Proteinkonzentration und anderen Zusätzen in der Testlösung abhängig ist.

Die Bestimmung des abgespaltenen Phosphates erfolgte nach der modifizierten Bastiaanse-Methode (3).

Die untersuchten Variationen der Meßbedingungen und der $\mathrm{Zu}$ sammensetzung der Testlösung werden bei den einzelnen Versuchen speziell erwähnt.

Die gereinigte Prostataphosphatase wurde mit $0,05 \mathrm{~mol} / \mathrm{l} \mathrm{Na}-$ triumacetatpuffer vom pH 5,5 entsprechend verdünnt.

\section{Resultate}

\section{Abhängigkeit der Farbintensität und der Farbstabilität des Phenolphthaleins vom $\mathrm{pH}$-Wert der Meßlösung} Die Farbintensität des Phenolphthaleins ( $\mathrm{pK}=9,2$ ) steigt vom $\mathrm{pH} 8,5$ bis 10 stark an und nimmt mit höheren pH-Werten nur noch wenig zu. Beim pH 10 besteht eine lineare Korrelation zwischen der Farbintensität und der Phenolphthaleinkonzentration (vgl. Abb. 2), zudem wird die Farbintensität von den Phosphationen (bis zu einer Konzentration von $0,5 \mathrm{~mol} / \mathrm{l}$ ) nicht beeinflußt.

Die Farbstabilität des Phenolphthaleins, die bis zum $\mathrm{pH} 10$ gegeben ist, nimmt mit steigendem $\mathrm{pH}$-Wert sehr rasch ab (Abb. 1). Aus diesem Grund wurde bei.den folgenden Versuchen die Farbintensität des Phenolphthaleins beim $\mathrm{pH} 10$ bestimmt.

\section{Abhängigkeit der Farbintensität des Phenolphthaleins von der Proteinkonzentration}

In Abbildung 2 ist die Abhängigkeit der Farbintensität sowohl von der Phenolphthalein- wie auch von der Albu-

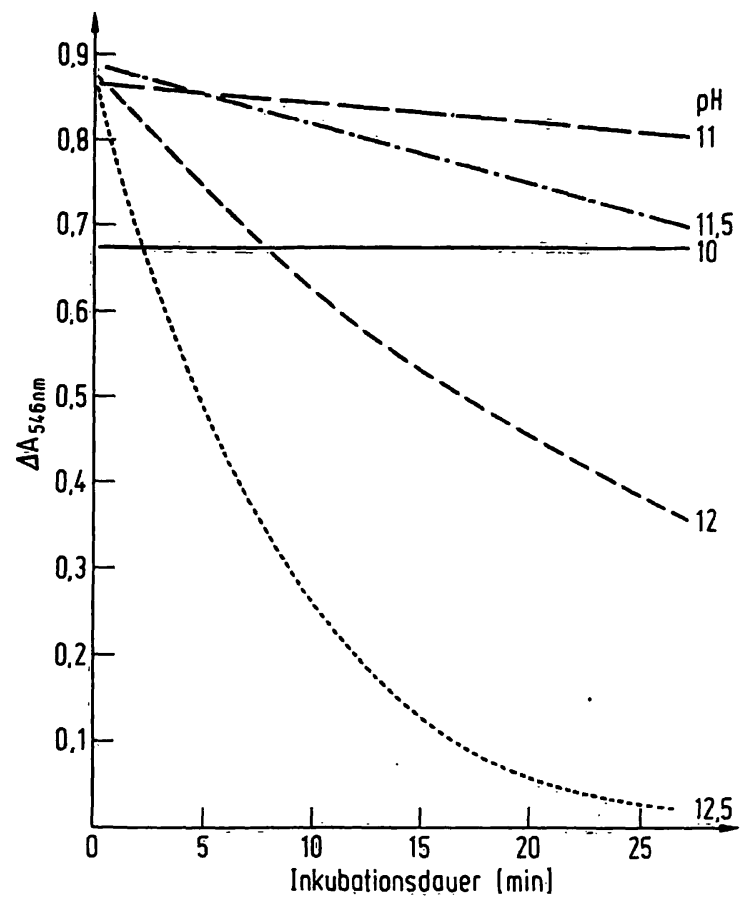

Abb. 1. Farbstabilität des Phenolphthaleins in Abhängigkeit vom pH-Wert.

$10 \mathrm{mg} / 1$ Phenolphthalein werden in $0,4 \mathrm{~mol} / 1$ Natriumphosphatpuffer der entsprechenden pH-Werte gelöst; bei $25^{\circ} \mathrm{C}$ wird während 30 Minuten die Absorption bei der Wellenlänge $546 \mathrm{~nm}$ laufend registriert.

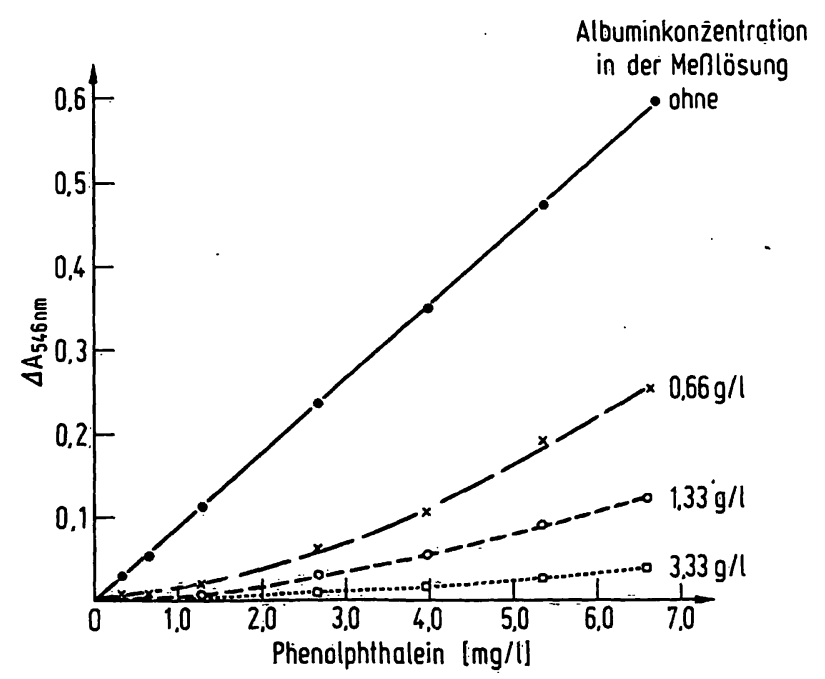

Abb. 2. Einfluß von Albumin auf die Farbintensität des Phenolphthaleins bei $\mathrm{pH} 10$.

Die angegebenen Mengen Phenolphthalein wurden in $0,4 \mathrm{~mol} / \mathrm{I}$ Natriumphosphatpuffer vom $\mathrm{pH} 10$, der unterschiedliche Albuminkonzentrationen enthielt, gelöst und die Absorption bei der Wellenlänge $546 \mathrm{~nm}$ bestimmt.

minkonzentration dargestellt. Im albuminfreien Ansatz entspricht die Farbreaktion dem Lambert-Beerschen Gesetz. Durch Zusatz von Albumin wird die Farbintensität wesentlich vermindert, wobei die prozentuale $A b$ sorptionsabnahme vom Verhältnis Phenolphthalein:Albumin abhängig ist. 
Der Einfluß des Albumins auf die Farbintensität des Phenolphthaleins ist $\mathrm{pH}$-abhängig, mit steigendem $\mathrm{pH}$ Wert wird dieser Einfluß geringer und hört über pH 11,5 auf.

\section{Einfluß von Alkohol auf die Farbintensität des Phenol- phthaleins}

Die Abbildung 3 zeigt ein typisches Beispiel für den Einfluß von Alkohol (1-Butanol) auf die Farbintensität des Phenolphthaleins in der albuminfreien sowie in der albuminhaltigen Meßlösung. Ohne Zusatz von Albumin wird die Farbintensität des Phenolphthaleins mit zunehmender Konzentration an 1-Butanol verringert.

Andererseits wird die durch 2,5 g/l Albumin verursachte Verminderung der Farbintensität durch Zusatz von 1-Butanol zum Teil aufgehoben. Die Absorption der Meßlösung, die $6,6 \mathrm{mg} / 1$ Phenolphthalein und 2,5 g/1 Albumin enthält, wird durch Zusatz von $0,4 \mathrm{~mol} / 1$ 1-Butanol um $820 \%$ erhöht. Dieser Alkoholeinfluß auf die Farbintensität des Phenolphthaleins ist abhängig von der Albumin-, Alkohol- und Phenolphthaleinkonzentration sowie vom pH-Wert der Meßlösung und dem verwendeten Alkohol. Unter sonst identischen Bedingungen wird eine gleiche Erhöhung der Absorption des Phenolphthaleins in der $2,5 \mathrm{~g} / 1$ Albumin-haltigen Meßlösung erreicht durch Zusatz von $5 \mathrm{~mol} / 1$ Methanol, $2 \mathrm{~mol} / 1$ Ethanol, 0,8 mol/1 1-Propanol, 0,4 mol/1 1-Butanol oder 0,1 mol/1 1-Pentanol. Dies muß berücksichtigt werden, wenn der Einfluß von Alkohol auf die Aktivität der Prostataphosphatase mit Phenolphthaleinmonophosphat als Substrat untersucht wird.

\section{Einfluß von Albumin auf die Michaelis-Konstante des Phenolphthalein-monophosphates sowie auf die pH-Aktivitätskurve der Prostataphosphatase}

In Abbildung 4 ist die Aktivität der Prostataphosphatase beim pH 5,5 in Abhängigkeit zur Konzentration an Phenolphthalein-monophosphat dargestellt. Im albuminfreien Testansatz wird die maximale Reaktionsgeschwindigkeit schon mit einer Substratkonzentration von $125 \mu \mathrm{mol} / 1$ erreicht. Mit $3 \mathrm{~g} / 1$ Albumin im Testansatz wird die wesentlich höhere maximale Reaktionsgeschwindigkeit erst mit einer Phenolphthalein-monophosphat-Konzentration erreicht, die über $1 \mathrm{mmol} / 1$ liegt.

Die Michaelis=Konstante des Phenolphthalein-monophosphates ist abhängig von der Albuminkonzentration im Testansatz. Zur Halbsättigung der Prostataphosphatase muß mit steigender Albuminkonzentration auch die Substratkonżentration erhöht werden.

In Abbildung 5 sind die pH-Aktivitätskurven der Prostataphosphatase bei einer gegebenen Substratkonzentration von $1,0 \mathrm{mmol} / 1$ Phenolphthalein-monophosphat in Abhängigkeit zur Albuminkonzentration in der Testlösung dargestellt. Unter diesen Testbedingungen liegt

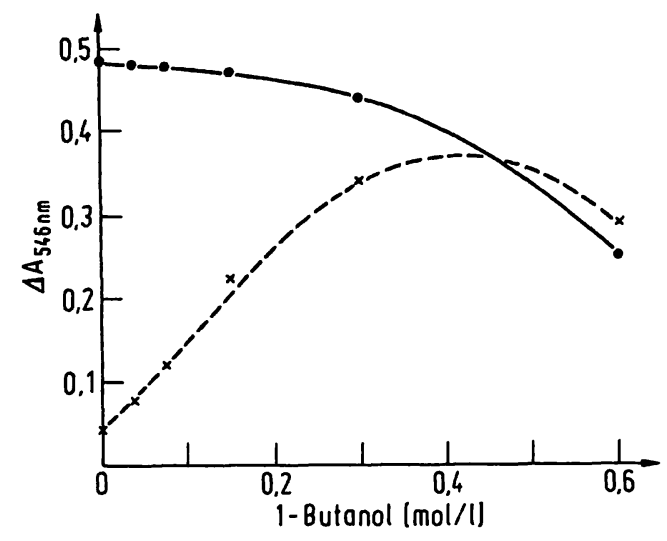

Abb. 3. Einfluß von 1-Butanol auf die Farbintensität des Phenolphthaleins in albuminfreier und albuminhaltiger Meßlösung.

$6,6 \mathrm{mg} / 1$ Phenolphthalein wurde in $0,4 \mathrm{~mol} / 1 \mathrm{Natrium}-$ phosphatpuffer vom pH 10 gelöst und die Absorption

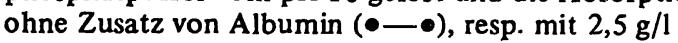
Albumin $(x-x)$ bei Anwesenheit der entsprechenden Konzentrationen an 1-Butanol bei der Wellenlänge 546 nm gemessen.

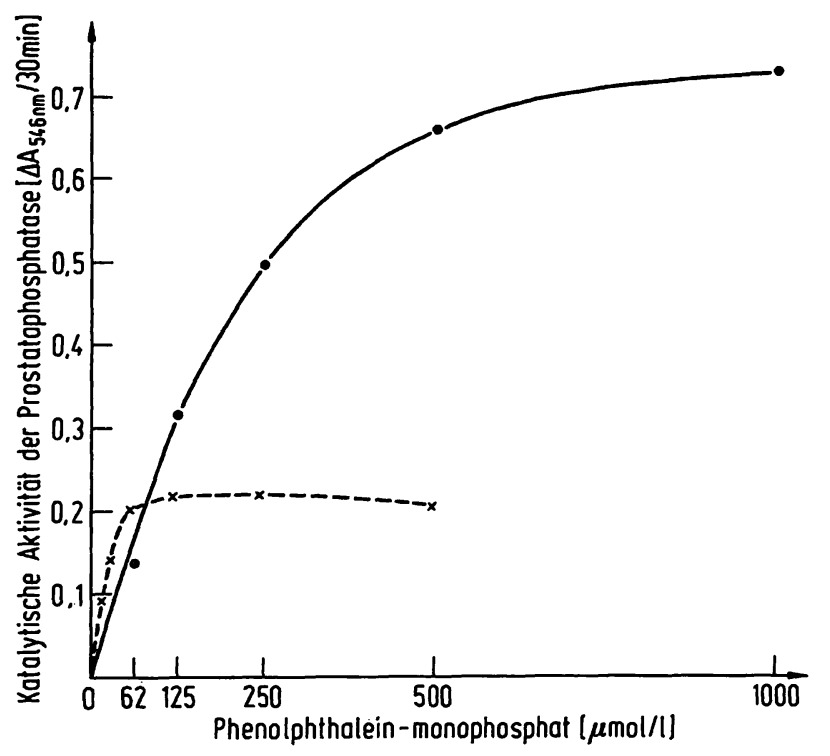

Abb. 4. Aktivität der Prostataphosphatase in Abhängigkeit zur Phenolphthaleinmonophosphat-Konzentration mit und ohne Albuminzusatz.

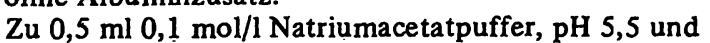
einer entsprechenden Konzentration an Phenolphthaleinmonophosphat ohne Zusatz von Albumin $(x-x)$, resp. mit $3 \mathrm{~g} / 1$ Albumin (•- $\bullet$ ) wurden $0,1 \mathrm{ml}$ Prostataphosphataselösung zugemischt, nach 30 Minuten Inkubation bei $37^{\circ} \mathrm{C} 2,5 \mathrm{ml} 0,4 \mathrm{~mol} / 1$ Natriumphosphatpuffer zum Abstoppen der Reaktion zugegeben und die Absorption des Phenolphthaleins bei der Wellenlänge $546 \mathrm{~nm}$ bestimmt.

das pH-Optimum des albuminfreien Ansatzes bei pH 7. Bei Anwesenheit von Albumin verschiedener Konzentration wird das $\mathrm{pH}$-Optimum nach 5,8 verschoben. Im alkalischen Teil der pH-Aktivitätskurve hat Albumin keinen oder nur einen hemmenden Einfluß auf die Prostataphosphatase, während im sauren Bereich durch 


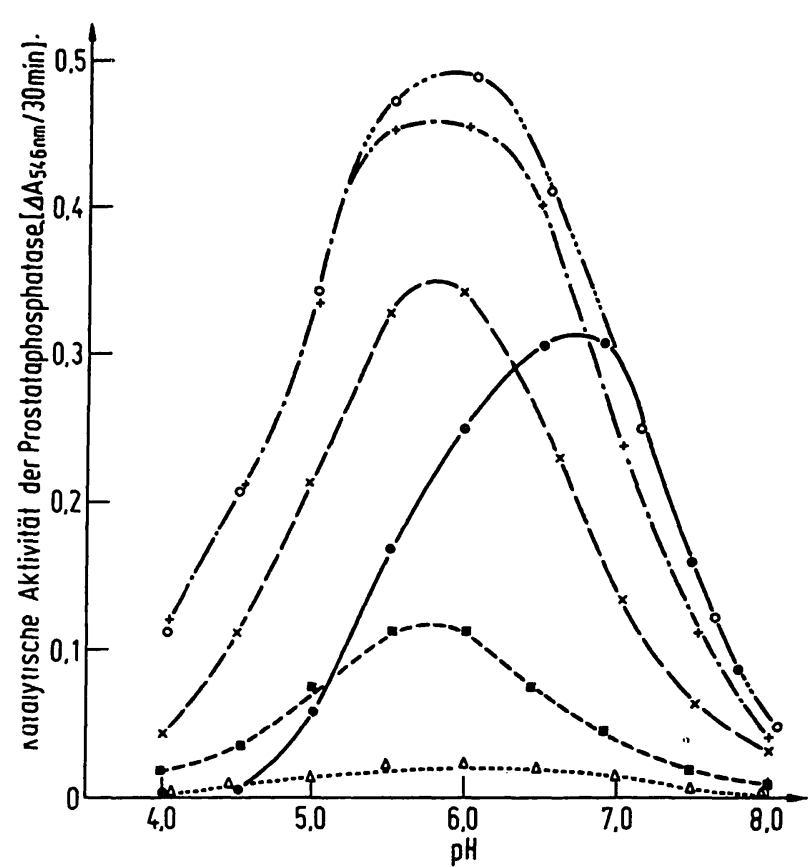

Abb. 5. pH-Aktivitätskurven der Prostataphosphatase in Abhängigkeit von der Albuminkonzentration im Testansatz. $\mathrm{Zu} 0,5 \mathrm{ml} \mathrm{0,05} \mathrm{mol} / 1$ Natriumacetat, $0,05 \mathrm{~mol} / 1$ TrisPuffer der entsprechenden pH-Werte und $1,0 \mathrm{mmol} / 1$ Phenolphthalein-monophosphat

$(\bullet-\bullet)$ ohne Zusatz von Albumin

$(0-\cdots-0)$ mit 1,55 g/l Albumin

(+-.-.++) mit 3,12 g/l Albumin

$(x-x)$ mit $6,25 \mathrm{~g} / 1$ Albumin

(m-..-) mit 12,5 g/l Albumin

$(\Delta \ldots . . . \Delta) \quad$ mit $25 \mathrm{~g} / 1$ Albumin

werden $0,1 \mathrm{ml}$ Prostataphosphataselösung zugemischt, 30 Minuten bei $37^{\circ} \mathrm{C}$ inkubiert und nach Abstoppen der Reaktion durch Zugabe von $2,5 \mathrm{ml} \mathrm{0,4} \mathrm{mol} / 1$ Natriumphosphatpuffer vom pH 10 die Absorption bei der Wellenlänge $546 \mathrm{~nm}$ gemessen.

eine Albuminzugabe, die in einem günstigen Konzentrationsverhältnis zum Phenolphthalein-monophosphat steht, eine wesentliche Erhöhung der ProstataphosphataseAktivität erreicht werden kann. Der Einfluß von Albumin auf die Aktivität der Prostataphosphatase ist außer von der Substratkonzentration auch vom $\mathrm{pH}-$ Wert abhängig.

Der Einfluß des Albumins auf die Prostataphosphataseaktivität ist nicht einfach durch eine das Enzym stabilisierende Wirkung während der Reaktion zu erklären, denn auch im albuminfreien Testansatz ergibt sich eine lineare Korrelation zwischen Farbintensität und enzymatischer Reaktionsdauer (0-60 min).

Die Abbildung 6 zeigt den Einfluß von unterschiedlicher Albuminkonzentration auf die Prostataphosphataseaktivität. Die Resultate sind in Prozent des albuminfreien Ansatzes ausgedrückt. Um den Einfluß des Albumins auf die Farbintensität des freigesetzten Phenolphthaleins auszuschalten, wurde parallel dazu auch das abgespaltene Phosphat bestimmt. Eine Aktivitätshemmung der Prostataphosphatase durch Albumin ist demnach nur durch die albuminbedingte AbsorptionsVerminderung des von der Phosphatase freigesetzten Phenolphthaleins vorgetäuscht.

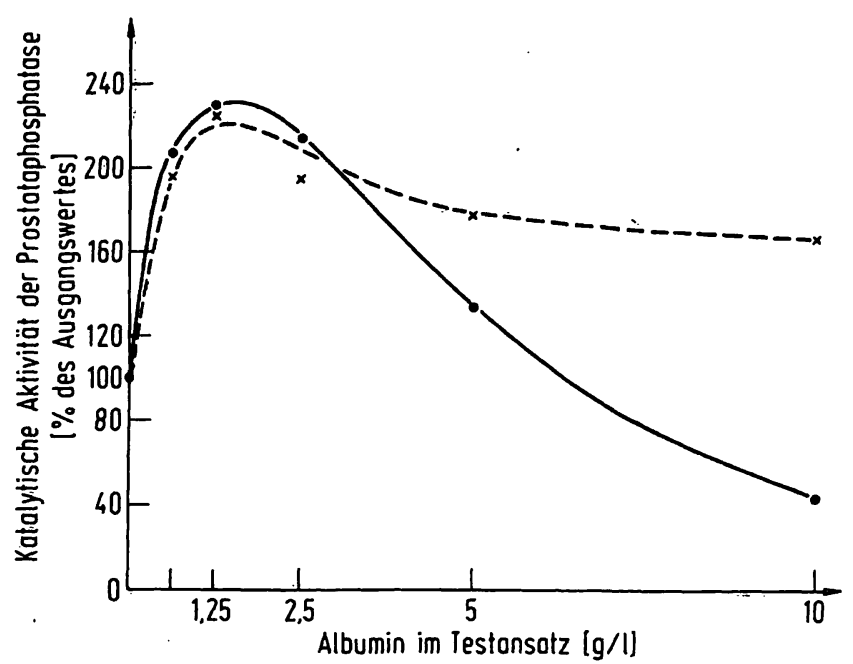

Abb. 6. Einfluß von Albumin auf die Aktivitätsbestimmung der Prostataphosphatase mit Phenolphthalein-monophosphat.

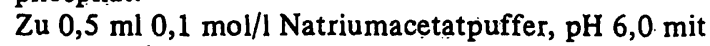
$0,8 \mathrm{mmol} / \mathrm{l}$ Phenolphthalein-monophosphat und einer entsprechenden Konzentration an Albumin werden $0,1 \mathrm{ml}$ Prostataphosphataselösung zugemischt, 30 Minuten bei $37^{\circ} \mathrm{C}$ inkubiert und anschließend in einem Teil der Inkubationslösung das abgespaltene Phosphat $(x-x)$ und

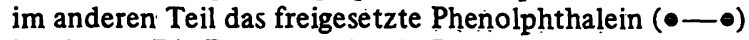
bestimmt. Die Resultate sind in Prozent des albuminfreien Ansatzes ausgedrückt.

\section{Einfluß verschiedener Alkohole auf die Aktivität der Prostataphosphatase, gemessen mit Phenolphthalein- monophosphat als Substrat}

Im albuminfreien Testansatz ist bei Anwesenheit von Alkohol eine deutliche Aktivierung der Prostataphosphatase festzustellen, die von der Art und der Konzentration des Alkohols abhängig ișt (Abb. 7). Dabei wird mit 0,4 mol/1 1-Butanol die höchste Aktivierung von 150\% erreicht. Hingegen fällt bei Anwesenheit von 2,2 g/l Albumin die alkoholbedingte Aktivierung der Prostataphosphatase bedeutend geringer aus, wobei durch eine höhere Konzentration an 1-Propanol oder 1-Butanol die Enzymaktivität sogar gehemmt wird (Abb. 8). Bei diesen Resultaten ist $z$ beachten, daß in der albuminfreien Meßlösung die Farbintensität des vorhandenen Phenolphthaleins durch Alkoholzusatz verringert, und daß anderseits die albumin-bedingte Farbunterdrückung durch Alkoholzusatz zum Teil aufgehoben wird (cf. Abb. 3).

Der Alkoholeinfluß auf die Aktivität der Prostataphosphatase ist abhängig vom $\mathrm{pH}$-Wert der Reaktionslösung. In Abbildung 9 sind die entsprechenden Resultate für 1-Butanol im alkalischen $\mathrm{pH}$-Bereich dargestellt. Wie das Albumin, so übt auch das 1-Butanol im alkalischen pHBereich eher einen hemmenden Einfluß auf die Prostataphosphatase aus, während im sauren pH-Aktivitätskurvenbereich eine von der 1-Butanolkonzentration und dem $\mathrm{pH}$ abhängige Aktivierung festzustellen ist. Wie mit Albumin wird das pH-Optimum auch mit 1-Butanol gegen den sauren $\mathrm{pH}$-Bereich verschoben. 


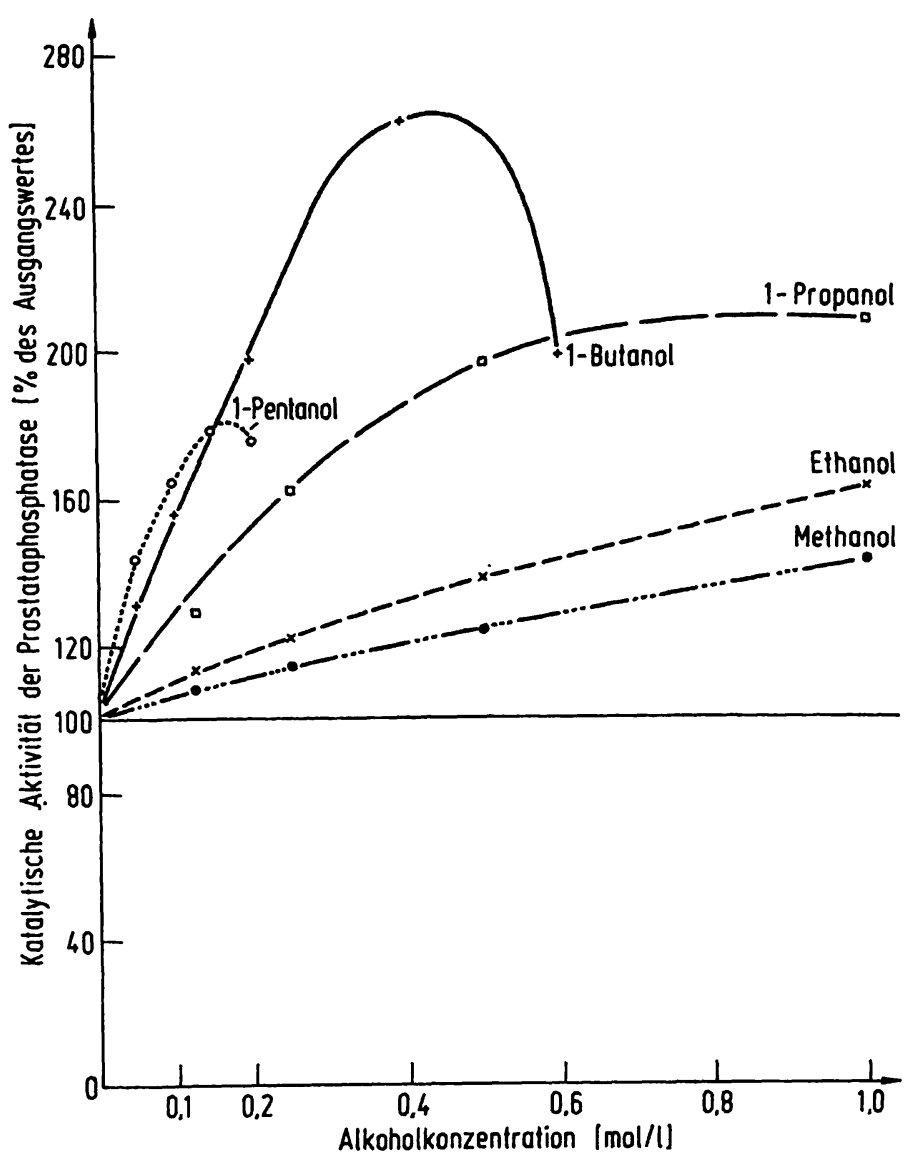

Abb. 7. Einfluß verschiedener Alkohole auf die Aktivität der Prostataphosphatase im albuminfreien Testansatz mit Phenolphthalein-monophosphat als Substrat.

$\mathrm{Zu} 0,5 \mathrm{ml} 0,1 \mathrm{~mol} / 1$ Natriumacetatpuffer, $\mathrm{pH} 5,5$ und $0,5 \mathrm{mmol} / 1$ Phenolphthalein-monophosphat mit den entsprechenden Konzentrationen der aufgeführten Alkohole werden $0,1 \mathrm{ml}$ Prostataphosphataselösung zugemischt, 30 Minuten bei $37^{\circ} \mathrm{C}$ inkubiert und nach Abstoppen der Reaktion durch Zugabe von $2,5 \mathrm{ml} \mathrm{0,4} \mathrm{mol} / 1$ Natriumphosphatpuffer vom pH 10 die Absorption des freigesetzten Phenolphthaleins bei der Wellenlänge $546 \mathrm{~nm}$ gemessen.

\section{Dialysierbarkeit des Phenolphthalein-monophosphates bei Anwesenheit von Albumin und 1-Butanol}

$\mathrm{Zu}$ diesem Versuch wurden $10 \mathrm{ml}$ einer $4 \mathrm{mmol} / 1$ Phenolphthalein-monophosphatlösung, gepuffert mit $0,1 \mathrm{~mol} / 1$ Natriumacetat vom $\mathrm{pH}$ 6,0 in êinen Dialyseschlauch gefuillt und bei $25^{\circ} \mathrm{C}$ während 3 Tagen gegen $100 \mathrm{ml}$ 0,1 mol/1 Natriumacetat, $\mathrm{pH} 6,0$ und $5000 \mathrm{U} / 1$ Prostataphosphatase dialysiert. Nach bestimmten Zeitintervallen wurde eine Probe der Diälysierlösung mit $0,4 \mathrm{~mol} / \mathrm{l}$ Natriumphosphatpuffer vom $\mathrm{pH} 10$ versetzt und die Abșorption des diffundierten und durch die Phosphatase freigesetzten Phenolphthaleins gemessen.

Das Phenolphthalein-monophosphat diffundiert ohne Zusatz wie auch bei Vorhandensein von 0,4 mol/1 1-Butanol schnell und vollständig. Nach 1 Tağ konnten $90 \%$ des eingesetzten Substrates als Phenolphthalein in der Dialyșierlösung nạchgewiesen werden. Wird aber im Dialysierschlauch zum Phenolphthalein-monophosphat $25 \mathrm{~g} / 1$ Albumin zugemischt, so diffundieren nur $40 \%$

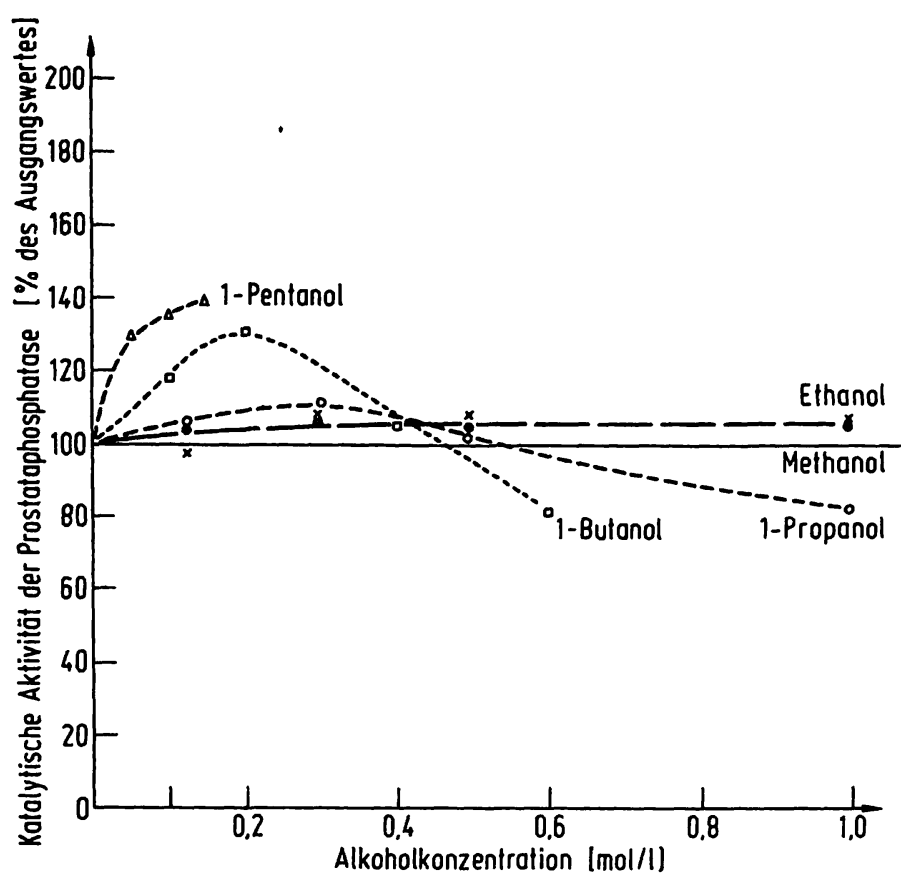

Abb. 8. Einfluß verschiedener Alkohole auf die Aktivität der Prostataphosphatase bei Anwesenheit von 2,2 g/l Albumin im Testansatz.

Bei gleichem Vorgehen wie für den Versuch der Abbildung 7 wurde der Testlösung 2,2 g/l Albumin zugesetzt.

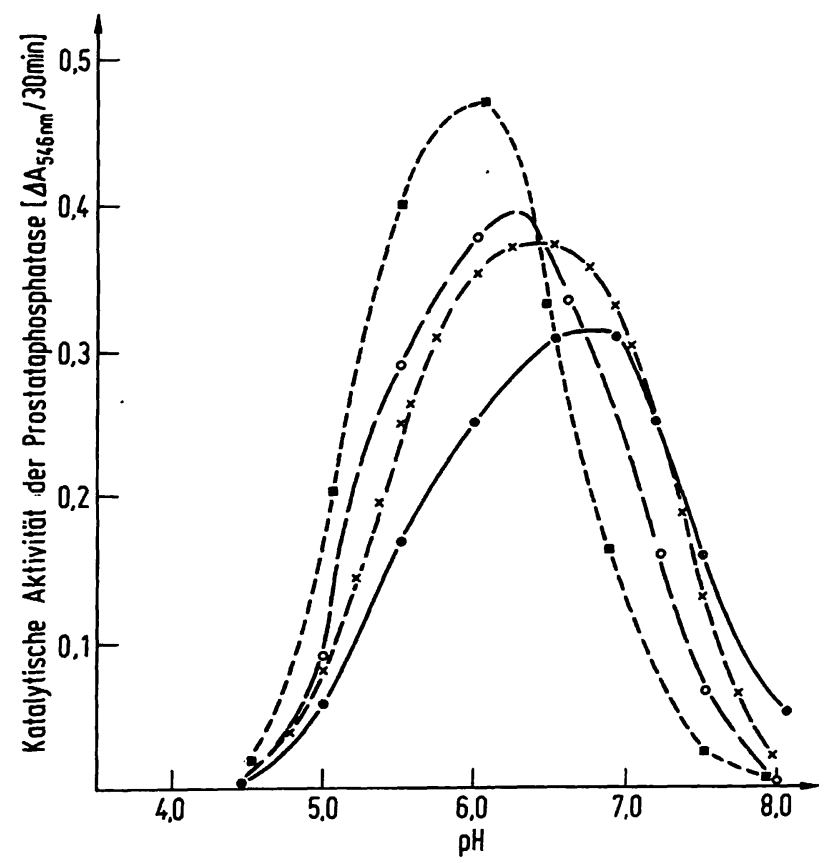

Abb. 9. pH-Aktivitätskurve der Prostataphosphatase in Abhängigkeit von der 1-Butanol-Konzentration im Testansatz. $\mathrm{Zu} 0,5 \mathrm{ml} \mathrm{0,05} \mathrm{mol} / 1 \mathrm{Natriumacetat}(\mathrm{pH} \mathrm{4,5-6,0),0,05}$ $\mathrm{mol} / \mathrm{l}$ Tris-Puffer (pH 6,0-8,0) der entsprechenden $\mathrm{pH}-$ Werte, mit 1,0 mmol/1 Phenolphthalein-monophosphat ohne Zusatz von 1 -Butanol $(\bullet-\bullet)$, mit $1,0 \mathrm{~mol} / 1$ 1Butanol (x-x), mit 0,2 mol/l 1-Butanol (0- - - $)$

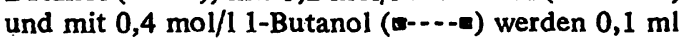
Prostataphosphataselösung zugemischt, 30 Minuten bei $37^{\circ} \mathrm{C}$ inkubiert und nach dem Abstoppen der Reaktion durch Zugabe von $2,5 \mathrm{ml} \mathrm{0,4} \mathrm{mol} / 1$ Natriumphosphatpuffer vom pH 10 die Absorption des freigesetzten Phenolphthaleins bei der Wellenlänge $546 \mathrm{~nm}$ bestimmt. 
des eingesetzten Substrates, während 60\% des Phenolphthalein-monophosphates vom Albumin im Dialysierschlauch zurückgehalten werden. Die Dialyse wird durch einen Zusatz von 0,4 mol/1 1-Butanol zur AlbuminPhenolphthalein-monophosphat-Mischung im Dialysierschlauch nicht wesentlich verbessert.

\section{Diskussion}

Auf Grund der mitgeteilten Resultate kann angenommen werden, daß Albumin im Meßbereich vom $\mathrm{pH} 10$ das Phenolphthalein bindet. Dadurch kann das Resonanzsystem dieses Säure-Base-Indikators nicht vollständig ausgebildet werden, was zu einer - von der Albuminkonzentration abhängigen - Verminderung der Farbintensität führt. Diese Bindung des Phenolphthaleins an Albumin scheint durch einen Zusatz von Alkohol zum Teil gelöst zu werden.

Auch das Phenolphthalein-monophosphat wird im pHBereich der enzymatischen Reaktion von Phosphatasen (pH 4-8) an Albumin gebunden. Dadurch wird die für die enzymatische Reaktion verfügbare Substratkonzentration in der Testlösung erniedrigt. Bei kleinen Phenolphthalein-monophosphat-Konzentrationen oder bei zu hohem Gehalt an Albumin bewirkt dies eine Verminderung der Prostataphosphatase-Aktivität, da zur Enzymsättigung nicht genügend freies Substrat vorhanden ist. Andererseits kann bei einem Überschuß an Phenolphthalein-monophosphat der Zusatz von Albumin die Substrathemmung vermindern oder gar aufheben.

Die Bindung des Chromophors und des Substrates an Albumin und die damit verbundene Beeinflussung der Aktivitätsbestimmung der Prostataphosphatase ist für das Penolphthalein-monophosphat nicht spezifisch. Sie tritt auch auf mit den Substraten Phenolphthaleindiphosphat (Sigma) und Thymolphthalein-mono- phosphat (Calbiochem). Beim Thymolphthalein-monophosphat sind die Verhältnisse allerdings insofern einfacher, da wegen der besseren Stabilität des Thymolphthaleins im stark alkalischen Milieu die Farbintensität des freigesetzten Thymolphthaleins beim $\mathrm{pH} 12$ bestimmt werden kann. Bei diesem $\mathrm{pH}$-Wert vermag das Albumin den Chromophoren nicht mehr zu binden, so daß die volle Farbintensität gemessen werden kann.

Die Aktivitätsbestimmung der Prostataphosphatase wird durch Albumin nicht beeinflußt, wenn Phenylphosphat und 4-Nitrophenylphosphat als Substrat eingesetzt werden.

Die in dieser Arbeit mitgeteilten Resultate machen es verständlich, daß mit Phenolphthaleinphosphat als Substrat unter bestimmten Testbedingungen nicht eine lineare, sondern eine parabolische Korrelation der eingesetzten katalytischen Konzentrationen zur gemessenen Farbintensität des Phenolphthaleins auftreten kann (4-9). Sie zeigen auch, daß die Erhöhung der Farbintensität um 90\%, wie sie Appleyard bei einem Zusatz von $15 \%$ Ethanol zum Testansatz bei der Aktivitätsbestimmung der Prostataphosphatase erreichte, nicht zum vornherein durch eine Alkoholaktivierung des Enzyms zu erklären ist (9). Vor allem aber machen diese Resultate klar, daß die Aktivitätsbestimmung der Prostataphosphatase mit Phenolphthaleinphosphat als Substrat von verschiedenen Faktoren dermaßen beeinflußt wird; daß die Interpretation der Ergebnisse schwierig und eine Berechnung der katalytischen Konżentrationen des Enzyms auf Grund der gemessenen Farbintensität des Phenolphthaleins häufig zu fehlerhaften Ërgebnissen führt.

\section{Dankșagung}

Der Autor dankt Frau $H$. Dettmar für die effiziente technische Hilfe bei der Durchführung der Versuche.

\section{Literatur}

1. Gallati, H. \& Roth, M. (1976), diese Z. 14, 581-587.

2. Bessey, O., Lowry, O. \& Brock, M. (1946), J. Biol. Chem $164,321-329$.

3. Bastiaanse, A. \& Meijers, C. (1968), diese Z. 6, 48-51.

4. Huggins, C. \& Talalay, P. (1945), J. Biol. Chem. 159, 399-410.

5. Linhardt, K. \& Walter, K. (1952), Hoppe-Seyler's Z. Physiol Chemie 289, 245-253.
6. Babson, A., Read, P. \& Phillips, G. (1959), Am. J. Clin. Pathol. $32,83-87$.

7. Klein, B., Read, P. \& Babson, R. (1960), Clin. Chim. 6, 269-275.

8. Rzhekhina, N. (1962), Biokhimiya 27, 359-365.

9. Appleyard, J. (1948), Biochem. J. 42, 596-597.

Dir. H. Gallati

Diagnostische Forschungsabteilung F. Hoffmann-La Roche \& Co. AG Grenzacherstraße 124 CH-4002 Basel 